\title{
Протезирование трехстворчатого клапана при удалении злокачественной опухоли правого желудочка сердца
}

\author{
Витовский Р. М., Исаенко В. В., Онищенко В. Ф., Пищурин А. А., Дядюн Д. Н., \\ Мартыщенко И.В.
}

ГУ «Национальный институт сердечно-сосудистой хирургии имени Н. М. Амосова НАМН» (Киев)

\begin{abstract}
Цель исследования - представить неординарный случай вторичного поражения правого желудочка (ПЖ) злокачественным новообразованием, характер и размер которого был распознан окончательно во время операции, а также особенности хирургического лечения такого поражения, которое сопровождалось протезированием трехстворчатого клапана.

Материалы и методы. В НИССХ имени Н. М. Амосова НАМН с 1970 г. по настоящее время было выполнено хирургическое лечение 62 больным с различными видами и локализацией злокачественных опухолей сердца (3ОС), что составило 6,8\% от 912 пациентов с сердечными опухолями.

Результаты. Представленное в работе клиническое наблюдение больного с вторичным опухолевым поражением правого желудочка (ПЖ) представляет практический интерес с точки зрения сложности диагностики, необычного пути метастазирования и особенностей хирургического лечения.

Выводы. Метастазы в сердце обычно возникают благодаря их гематогенному или лимфогенному распространению или вследствие прямой инвазии. Злокачественные опухоли сердца могут не только поражать его стенки, но и распространяться на его клапанные структуры, что требует коррекции клапанных поражений. Нельзя исключить наличие тромбов в камерах сердца, учитывая нарушение гемодинамики, связанное с наличием новообразования.
\end{abstract}

Ключевые слова: злокачественные опухоли, метастазы, инвазия.

Метастазирование в сердце или прорастание опухоли в миокард и перикард регистрируется, по различным данным, у 0,3-27\% умерших от злокачественных новообразований. Метастазы в сердце чаще всего возникают на фоне развернутой клинической картины основного заболевания, обычно имеется первичное или метастатическое поражение где-либо в грудной полости. Вторичные опухоли сердца встречаются в $13-40$ раз чаще, чем первичные [2, 4]. Наиболее часто такое вторичное поражение наблюдается при раке легкого, пищевода, молочной или щитовидной железы, а также при меланомах, лимфомах, миеломах, лимфогранулематозе [68]. В абсолютных цифрах метастазы в сердце чаще всего встречаются при раке молочной железы и легких, что отражает наибольшую распространенность этих опухолей. Тем не менее иногда метастазы в сердце могут быть первыми проявлениями опухоли иной локализации.

В современной кардиохирургической литературе до $75 \%$ всех первичных сердечных новообразований разделяют по гистологическому строению на доброкачественные, $25 \%$ относят к первичным злокачественным неоплазмам $[2,3,7]$. Также опухоли сердца подразделяют на первичные доброкачественные и злокачественные новообразования и вторичные (метастатические) злокачественные опухоли [1-5].
Первичные злокачественные сердечные новообразования встречаются крайне редко, что объясняется, по-видимому, особенностями метаболизма миокарда, коронарным кровотоком и ограниченностью лимфатических соединений внутри сердца [2]. На аутопсии они выявляются намного чаще, чем при клинических исследованиях.

Как и при первичных опухолях сердца, клинические проявления вторичных опухолей больше зависят от локализации и размеров опухоли, чем от ее гистологического типа. Метастазы не служат причиной существующих клинических проявлений у большинства больных, а возникают на фоне предшествующих проявлений злокачественной опухоли. Метастазы в сердце могут давать различные симптомы, чаще всего это одышка, появление систолического шума, признаки острого перикардита, тампонада сердца, быстрое увеличение площади сердечного контура при рентгенологическом исследовании, вновь появившиеся нарушения ритма сердца, атриовентрикулярная блокада, застойная сердечная недостаточность. Многие из этих признаков и симптомов могут наблюдаться также при миокардитах, перикардитах, кардиомиопатиях либо быть результатом лучевой терапии или химиотерапии [2-10]. Ангиография может выявить форму отдельных 
частей опухоли. У большинства больных с метастазами в сердце болезнь имеет достаточную распространенность, часто нарушающую гемодинамику [2].

Хирургическое лечение злокачественных опухолей сердца (3ОС) в большинстве случаев малоэффективно и пока не вносит принципиальных изменений в прогноз больных с этой патологией. Главным объяснением тому до сих пор остается поздняя диагностика заболевания [2-4]. Поэтому сегодня клиницисты вынуждены ограничиваться паллиативными хирургическими операциями, сочетая их в послеоперационном периоде с лекарственной и лучевой терапией. Некоторым кардиохирургам удавалось с помощью такого подхода продлить жизнь оперированных пациентов с первичными злокачественными новообразованиями сердца до 3-4,5 лет.

В настоящее время многие кардиохирурги ведущих клиник мира видят выход из создавшегося столь серьезного положения в ранней дифференциальной диагностике злокачественного опухолевого роста, а также в безотлагательной пересадке сердца, что позволяет добиться значительно лучших результатов.

Поздняя постановка диагноза не редкость в диагностике злокачественной опухоли сердца. Клинические проявления часто начинают появляться при значительном опухолевом поражении сердца и могут крайне быстро прогрессировать. Об этом свидетельствует клинический случай, который мы хотим представить в данной работе.

Цель исследования - представить неординарный случай вторичного поражения правого желудочка злокачественным новообразованием, характер и размер которого был распознан окончательно во время операции, а также особенности хирургического лечения такого поражения, которое сопровождалось протезированием трехстворчатого клапана.

Материалы и методы. По данным НИССХ имени Н. М. Амосова НАМН, с 1970 г. по настоящее время в Институте наблюдалось 62 больных с различными видами и локализацией злокачественных опухолей сердца, у которых выполнялось хирургическое лечение, что составило 6,8\% от общего количества наблюдаемых пациентов с сердечными опухолями (912). Локализация ЗОС в правом желудочке (ПЖ) отмечалась у $5(8,1 \%)$ больных, что является самой редкой по частоте локализации изолированного выявления в камерах сердца данной патологии (наиболее часто - в левом предсердии (ЛП) - 24, что составило $38,7 \%$ ).

Учитывая изложенное, мы сочли целесообразным представить одно из наших наблюдений опухолевого поражения правого желудочка метастазом светлоклеточного рака почки. Этот случай интересен в связи со сложностью диагностики опухолевого процесса, крайне редким направлением метастазирования, недиагностированным объемом поражения сердца, а также непредсказуемым объемом хирургического лечения.

Изучались особенности клинического течения заболевания. Применялись как лабораторные, так и инструментальные методы исследования, среди которых, наряду с ЭКГ, ЭхоКГ, применялись КТ и МРТ, позволившие выявить точную локализацию новообразования.

Результаты. Пациент К., 55 лет (и/б № 2349), поступил 11.05.2017 г. в Национальный институт сердечно-сосудистой хирургии имени Н. М. Амосова НАМН (ИССХ) с диагнозом - опухоль правого желудочка (ПЖ). Из анамнеза было известно, что больному была выполнена частичная резекция левой почки (по поводу светлоклеточного рака почки) 02.12. 2016.

Через 5 месяцев пациент обратился к кардиологу по месту жительства с жалобами на одышку и общую слабость и был направлен на эхокардиографическое исследование, при проведении которого в полости ПЖ было обнаружено малоподвижное новообразование, заполняющее большую часть его полости. В связи с этим для уточнения диагноза и проведения предварительной кардиохирургической операции пациент был направлен в НИССХ.

Частота сердечных сокращений составляла 84 удара в 1 минуту. У пациента выслушивался небольшой интенсивности систолический шум в проекции верхушки сердца. Общий анализ крови определил: гемоглобин 115 г/л, эритроциты - 4,8 г/л, СОЭ - 9 мм/ч, лейкоциты - 14,2 г/л (лейкоцитарная формула - в пределах нормы: Эо-4, Пал-5, С-69, Лф-19, М-3). Рентгенографическое исследование выявило незначительное увеличение сердца, кардиоторакальный индекс составлял 0,45 . Каких-либо выраженных отклонений со стороны контуров сердца не наблюдалось. Патологических образований в легких не выявлено.

При поступлении в НИССХ при объективном обследовании обращали на себя внимание выраженная общая слабость, бледность кожных покровов, боли в области сердца. При осмотре - умеренные отеки нижних конечностей, при пальпации отмечено увеличение печени, выступающей ниже реберной дуги на 2 см, кожные покровы бледные, отмечался небольшой цианоз губ, пациент пониженного питания. Отмечались постоянные жалобы на выраженную одышку и утомляемость, связанные с физической нагрузкой, периодически возникающие боли в области сердца. АД - 130/80. Семейный анамнез, анамнез жизни - без особенностей. Из анамнеза стало известно, что при обследовании больного во время подготовки к операции частичной резекции левой почки больному проводилось эхокардиографическое исследование, которое показало наличие в полости ПЖ небольшого новообразования в области его верхушки, плотно сращенного со стенкой ПЖ. Однако на тот момент консультация кардиохирурга не прово- 


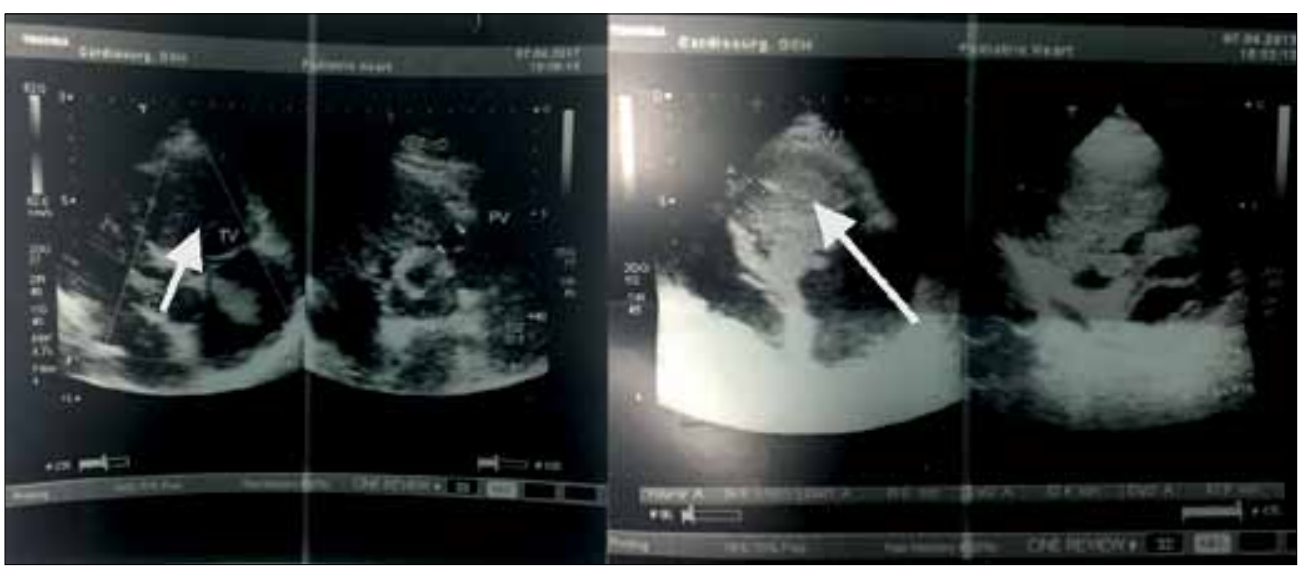

Рис. 1. Данные ЭхоКГ-исследования (стрелкой указано новообразование в полости ПЖ)

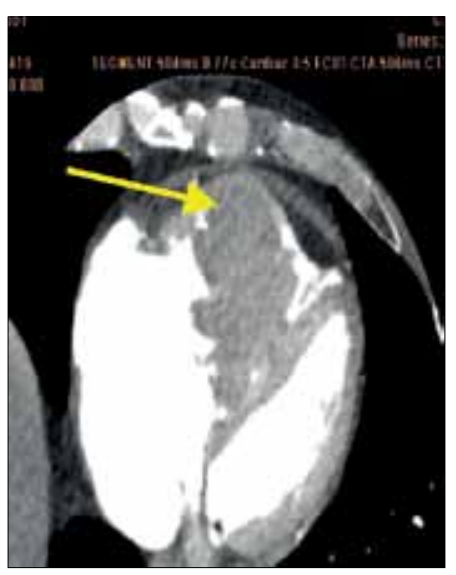

Рис.2. Данные КТ исследования: стрелкой указано новообразование в полости правого желудочка дилась. Этот факт может свидетельствовать о том, что новообразование в полости сердца уже могло быть метастазом рака почки, что требовало последующего регулярного контроля за его возможным ростом.

Со стороны других органов и систем, а также результатов лабораторных исследований патологических изменений не отмечено. Аускультативно определяется систолический шум малой интенсивности в проекции трехстворчатого клапана. Рентгенологическое исследование выявило незначительное увеличение сердца, которое имело митральную конфигурацию, отмечалось некоторое ослабление легочного рисунка. На ЭКГ - полный блок правой ножки пуска Гиса, частота пульса 80 ударов в 1 мин. Признаки умеренной гипертрофии обоих желудочков сердца, признаков коронарной недостаточности не выявлено. Общие клинические анализы крови и мочи были в пределах нормы, биохимический анализ крови: мочевина -8 , креатинин - 110 ммоль/л.

В условиях НИССХ при поступлении пациенту было произведено ЭхоКГ-обследование, при котором в полости ПЖ выявлено гроздевидное малоподвижное образование 7,6Ч6 см, плотно сращенное с межпредсердной перегородкой, пролабирующее в выходной тракт ПЖ с обструкцией последнего ( $\Delta \mathrm{P}>28$ мм рт. ст. $)$, небольшая трехстворчатая недостаточность (рис. 1) Обратный ток на трехстворчатом клапане определялся как небольшой. Фракция выброса левого желудочка составляла 68\%. Небольшое количество жидкости в полости перикарда. Таким образом, результаты ЭхоКГ-исследования свидетельствовали о том, что в данном случае речь идет о возможности злокачественного поражения сердца.

С учетом подозрения на злокачественный характер поражения сердца, для уточнения распространенности опухолевого процесса больному проведена спиральная компьютерная томография. При проведении КТ в полости правого желудочка было выявлено образование неправильной формы с бугристым контуром, четкими краями, условными размерами $72 \times 58 \times 65$ мм, объемом до 110 мл (рис. 2).

Папиллярно-трабекуллярный аппарат ТК интимно связан с образованием. Контур опухоли заполнял большую часть полости ПЖ, при этом частично перекрывая выходной тракт. Отсутствовали признаки значительных стенозов и атеросклеротических бляшек коронарных артерий.

Магнитно-резонансная томография дала дополнительную информацию о распространении опухолевого процесса. Опухоль ПЖ, с внутриполостным характером роста, четкими бугристыми контурами, дольчатого строения, размерами 80Ч63Ч69 мм; субтотально заполняет полость, прилежит к нижней стенке ПЖ, правым отделам МЖП (апикальная часть), свободной стенке ПЖ (средние, апикальные отделы), распространяется в область выходного тракта, создавая его обструкцию. Образование интимно прилежит к задним отделам фиброзного кольца ТК, протяженностью до 23 мм, частично сращено со створками ТК. Таким образом, наряду с поражением стенки ПЖ в опухолевый процесс был значительно вовлечен клапанный аппарат трехстворчатого клапана (ТК) с частичным поражением фиброзного кольца, что до некоторой степени вызывало сомнение в возможности выполнения протезирования ТК после обширного удаления опухоли с иссечением клапанного аппарата. Следует отметить, что при КТ и МРТ в полости правого предсердия каких-либо новообразований не выявлено.

При проведении ФЭГДС были выявлены эрозии антрального отдела желудка, в связи с чем проведена 
соответствующая успешная терапия выявленных эрозий, однако хирургическое лечение на этот период было отложено.

При очередном эхокардиографическом контроле 07.06.17 г. в полости правого предсердия был обнаружен тромб размером до 3 см без признаков его фиксации.

Учитывая значительный риск полной обструкции правого атриовентрикулярного отверстия, фрагментации новообразования и развития эмболических осложнений, наличие свободного тромба в полости правого предсердия, больному предложено хирургическое лечение, которое предполагало освобождение полости ПП от опухолевой ткани и протезирование трехстворчатого клапана и было проведено 09.06.2017 г.

При ревизии сердца выявлено значительное увеличение правого предсердия. При этом его стенка крайне плотная. Ушко левого предсердия увеличено и пальпаторно наполнено плотным содержимым. Стенка ПП также имеет повышенную плотность. В связи с крайней нетипичностью анатомии ПП подключение искусственного кровообращения имело некоторые особенности. После канюляции аорты верхняя полая вена канюлирована непосредственно в ее стенку канюлей «pacific». Нижняя полая вена канюлирована после начала искусственного кровообращения, вскрытия правого предсердия и визуальной ревизии его полости с использованием отсосов аппарата искусственного кровообращения. Тромбы, обнаруженные в полости ПП в большом количестве, препятствовали адекватной канюляции НПВ. После частичного их удаления удалось безопасно ввести канюлю в просвет НПВ и обжать ее тесемкой.

Операция выполнялась при умеренной гемодилюции и гипотермии $\left(28^{\circ} \mathrm{C}\right)$. Защита миокарда включала в себя применение раствора Кустодиол и местное охлаждение сердца ледяной крошкой.
После продольного рассечения ПП в его полости обнаружено значительное количество пристеночных тромбов (объемом до 120 мл), плотно прилегающих практически ко всем стенкам, прочно связанных с трабекулами, а также свободно плавающий овоидный тромб размером 4х3х3 см, представленный на рис. 3 .

Ушко ПП также полностью было заполнено тромботическими массами. Тромбы были удалены, и по мере возможности очищены межтрабекулярные пространства.

В полости ПЖ обнаружено белесоватое образование плотноэластической консистенции и неровной формы 7×6×6 см (рис. 4).

Опухоль была сращена со створками трехстворчатого клапана и подклапанным аппаратом и практически заполняла просвет ПЖ, фиксируясь к стенке ПЖ в области его верхушки и межжелудочковой перегородки. Острым и тупым путем фрагментами опухоль была удалена вместе со створками и папиллярными мышцами трехстворчатого клапана. Полость ПЖ практически полностью освобождена от опухолевой ткани. Основание ее на межжелудочковой перегородке было обработано диатермокоагуляцией. После многократного промывания правых отделов сердца следующим этапом было выполнено протезирования трехстворчатого клапана протезом ATS-29 (рис. 5).

После герметизации сердца и гемостаза была возобновлена сердечная деятельность. Из правой плевральной полости было эвакуировано 1100 мл светло-желтого экссудата. Таким образом, объем хирургического лечения заключался в удалении недиагностированных до операции тромбов ПП, удалении опухоли ПЖ и протезировании трехстворчатого клапана. Удаленные ткани отданы на гистологическое исследование.

При проведении гистологического исследования удаленного новообразования возникла необходимость

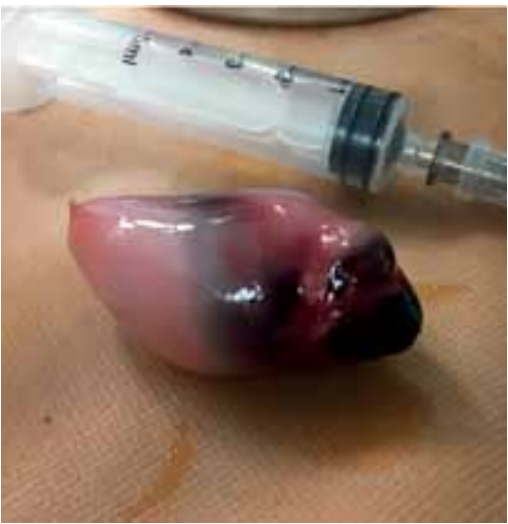

Рис. 3. Свободно плавающий овоидный тромб, удаленный из ПП

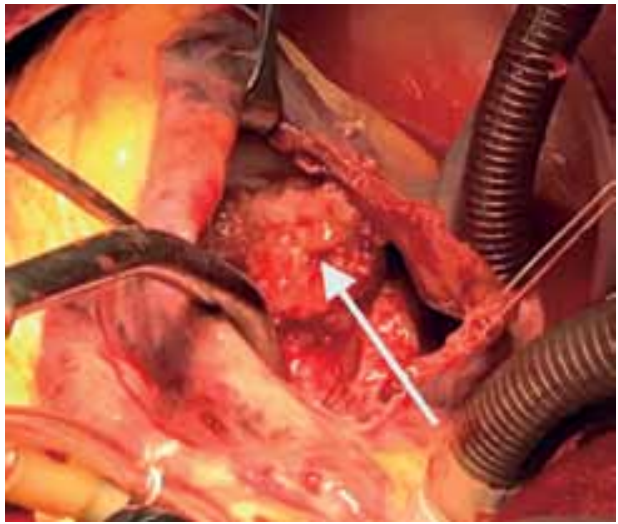

Рис.4. Опухоль правого желудочка указана стрелкой. Створки трехстворчатого клапана отведены крючками

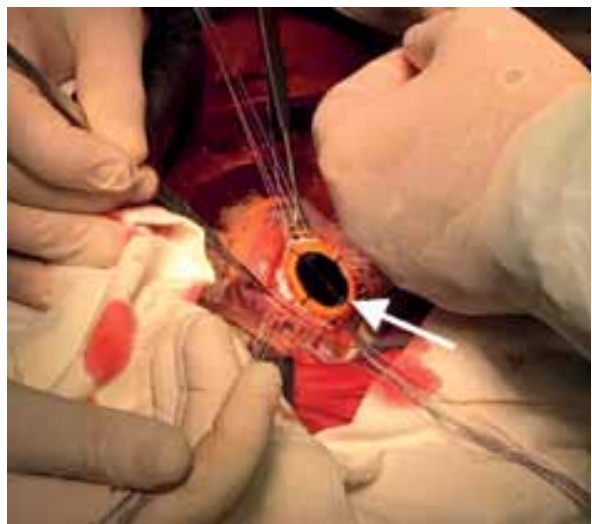

Puс 5. Протезирование трехстворчатого клапана после удаления опухоли 
выполнения иммуногистохимического исследования для уточнения диагноза, которое было произведено в Киевском городском клиническом онкоцентре. Согласно заключению, в исследованном материале был метастаз светлоклеточной карциномы почки.

В послеоперационном периоде пациент был экстубирован через 10 часов, наблюдалась умеренная сердечная недостаточность, потребовавшая применения в течение 72 часов допмина в дозе 5 мкг/кг/мин. в условиях реанимационного отделения. Необходимо отметить повышенную кровоточивость в послеоперационном периоде, вероятно связанную с анемией $(\mathrm{Hb}=86$ г/л) и интоксикацией, обусловленной основным заболеванием, что потребовало проведения 14.06.2017 г. левосторонней торакотомии без выявления явных источников кровотечения. В общеклиническое отделение пациент был переведен на 21 сутки.

В течение этого времени неоднократно возникала необходимость проведения дезинтоксикационной и обезболивающей терапии, которая продолжалась до выписки из кардиохирургического стационара. В удовлетворительном состоянии пациент был выписан на 33 сутки после операции и направлен на дальнейшее лечение в онкологический стационар по месту жительства.

Результаты и обсуждение. Представленный случай является неординарным примером вторичного поражения злокачественным опухолевым процессом ПЖ сердца. При проведении ЭхоКГ в полости ПП был выявлен плавающий тромб, который не мог переместиться в полость ПЖ, поскольку полость ПЖ была заполнена опухолью. Следует обратить внимание, что при ЭхоКГ не были выявлены пристеночные тромбы в ПП. Образование тромбов в ПП могло быть обусловлено нарушением гемодинамики в полостях правых отделов сердца злокачественной опухолью ПЖ. После проведения исследования было выполнено хирургическое лечение: удаление тромбов из ПП, удаление злокачественной опухоли ПЖ, а также выполнено протезирование трехстворчатого клапана механическим протезом ATS-29.

В описанном клиническом случае первичный очаг злокачественного поражения располагался в левой почке. Предположить метастазирование опухоли в сердце было возможно, учитывая анамнез заболевания.

\section{Выводы}

- Одним из вариантов распространения светлоклеточного рака почки может быть метастазирование в сердце.
- Метастазы в сердце обычно возникают благодаря их гематогенному или лимфогенному распространению или вследствие прямой инвазии. Клинические проявления на ранних стадиях развития опухолей сердца могут быть весьма скудными, поэтому необходимо применение ЭхоКГ при злокачественных заболеваниях, что обусловлено клинической настороженностью при данной патологии.

- Злокачественные опухоли сердца могут не только поражать его стенки, но и распространяться на его клапанные структуры, что требует коррекции клапанных поражений. Нельзя исключить наличие тромбов в камерах сердца, учитывая нарушение гемодинамики, связанное с наличием новообразования.

\section{Литература}

1. Г. В. Кнышов, Р. М. Витовский, В. П. Захарова. Опухоли сердца. - К., 2005. - 256 с.

2. Primary cardiac tumors on the verge of oblivion: a European experience over 15 years / Habertheuer A., Laufer G., Wiedemann D. et al. // J Cardiothorac Surg. - 2015. Vol. 10. - P. 56.

3. Incidence rate of primary cardiac tumors: a 14-year population study / Cresti A., Chiavarelli M., Glauber M. et al. // J Cardiovasc Med (Hagerstown). - 2016. - Vol. 17. P. 37-43.

4. Primary malignancies of the heart and pericardium / Burazor I., Aviel-Ronen S., Imazio M. et al. // Clin Cardiol. - 2014. - Vol. 37. - P. 582-8.

5. Factors affecting in-hospital mortality and likelihood of undergoing surgical resection in patients with primary cardiac tumors / Isogai T., Yasunaga H., Matsui H. et al. // J Cardiol. - 2016. - Vol. 10. - P. 1016.

6. Primary cardiac tumors: 32 years of experience from a Spanish tertiary surgical center / Barreiro M., Renilla A., Jimenez J. M. et al. // Cardiovasc Pathol. - 2013. - Vol. 22. - P. 424-7.

7. Surgery for primary cardiac tumors in children: early and late results in a multicenter European Congenital Heart Surgeons Association study / Padalino M. A., Vida V. L., Boccuzzo G. et al. // Circulation. - 2012. - Vol. 126. P. $22-30$.

8. Dias R. R., Fernandes F., Ramires F. J. et al. // Mortality and embolic potential of cardiac tumors / Arq Bras Cardiol. - 2014. - Vol. 103. - P. 13-8.

9. Clinicopathological and surgical experience with primary cardiac tumors / Barnes H., Conaglen P., Russell P. et al. // Asian Cardiovasc Thorac Ann. - 2014. - Vol. 22. P. 1054-8.

10. Bruce C. J. Cardiac tumours: diagnosis and management / C. J. Bruce // Heart. - 2011. - Vol. 97, № 2. - P. 151-160. 


\section{The replacement of the tricuspid valve in the removal of the malignant tumor of the right ventricle}

Vitovskiy R. M., Isaienko V. V., Pishchurin O. A., Dyadyun D. N., Onishchenko V. F., Martyshchenko I. V.

National M. M. Amosov Institute of Cardiovascular Surgery National Academy of Medical Sciences of Ukraine (Kyiv)

The aim of the study was to present an unusual case of secondary lesion of right ventricular (RV) with a malignant neoplasm, the character and size of which was finally recognized during the operation, as well as the peculiarities of surgical treatment of such lesion, which was accompanied by prosthetics of the tricuspid valve.

Material and methods. In the National Institute of Cardio-Vascular surgery named N.M. Amosov of the Academy of Medical Sciences of Ukraine since 1970 surgical treatment of 62 patients with various types and localization of malignant heart tumors was performed, which amounted to $6.8 \%$ of 912 patients with cardiac tumors.

Results. The clinical observation presented by the patient with secondary tumor's lesion of right ventricular (RV) is of practical interest from the point of view of the complexity of diagnosis, the unusual path of metastasis and the peculiarities of surgical treatment.

Conclusions. Metastases in the heart usually arise due to their hematogenous or lymphogenous spread or due to direct invasion. Malignant tumors of the heart can affect not only its walls, but also spread to its valve structures, which requires correction of valvular lesions. It is impossible to exclude the presence of thrombus in the chambers of the heart, considering the violation of hemodynamics associated with the presence of tumors.

Key words: malignant tumors, metastasis, invasion. 